\title{
Desempeño de los egresados desde la percepción de los empleadores
}

\section{Performance of graduates from the perception of employers}

DOI: http://dx.doi.org/10.17981/cultedusoc.12.1.2021.07

Recibido: 4 de febrero de 2020 Aceptado: 21 de mayo de 2020 Publicado: 1 de diciembre de 2020

Andrés Correal-Cuervo

Universidad de Boyacá. Tunja (Colombia)

sacorreal@uniboyaca.edu.co

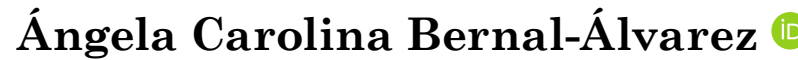

Universidad de Boyacá. Tunja (Colombia) angbernal@uniboyaca.edu.co

Juan Sebastián Cely-Bottía

Universidad de Boyacá. Tunja (Colombia) jscely@uniboyaca.edu.co

Cristian Alejandro Aguilar-Tovar

Universidad de Boyacá. Tunja (Colombia) caaguilar@uniboyaca.edu.co

Sandra Patricia Corredor-Gamba

Universidad de Boyacá. Tunja (Colombia) sancorredor@uniboyaca.edu.co

Para citar este artículo:

Correal-Cuervo, A., Bernal-Álvarez, Á., Cely-Bottía, J., Aguilar-Tovar, C. y Corredor-Gamba, S. (2021). Desempeño de los egresados desde la percepción de los empleadores. Cultura, Educación y Sociedad, 12(1), 103-118. DOI: http://dx.doi.org/10.17981/ cultedusoc.12.1.2021.07

Resumen

Las Instituciones de Educación Superior evalúan la congruencia educativa de acuerdo con las demandas del mercado laboral, garantizando condiciones acordes al contexto en inserción, desarrollo y desempeño profesional. Desde este referente el artículo tiene como objetivo analizar la percepción de empleadores con referencia al desempeño de egresados de la Universidad de Boyacá, en Tunja-Colombia. Es un estudio prospectivo transversal, con diseño descriptivo y fase correlacional, a través del muestreo probabilístico se obtuvo una muestra de 188 empresas; se entrevistaron empleadores de orden local, regional, nacional e internacional durante el período 2018-2019. Las variables estudiadas fueron competencias genéricas, formación y desempeño. Los principales resultados destacan la percepción de los empleadores cuando consideran que los egresados tienen un nivel alto con relación a la formación y desempeño profesional, así mismo, frente a las competencias generales resaltan la comunicación de pensamientos, ideas e información por escrito; se infiere que los egresados ponen en práctica habilidades comunicacionales asertivas; finalmente, se evidencia relación estadísticamente significativa entre formación y desempeño. Se concluye que el nivel de desempeño es acorde con el perfil profesional, evidenciando que son competentes en cualquier escenario en relación directa a referentes personales, profesionales y sociales. Palabras clave: Formación universitaria; egresados; empleadores; competencias profesionales

\section{Abstract}

Higher education institutions evaluate educational congruence according to the demands of the labor market, guaranteeing conditions according to the context in isertion, development and professional performance. From this point of reference, the article aims to analyze the perception of employers with reference to the performance of graduates of the University of Boyacá, in Tunja-Colombia. It is a prospective cross-sectional study, with a descriptive design and correlation phase. Through probabilistic sampling, a sample of 188 companies was obtained: Local, regional, national and international employers were interviewed during the 20182019 period. The variables studied were generic skills, training and performance. The main results highlight the perception of employers when they consider that graduates have a high level in relation to training and professional performance, likewise, compared to general competences, they highlight the communication of thoughts, ideas and information in writing: It is inferred that graduates put into practice assertive communication skills; finally, there is a statistically significant relationship between training and performance. It is concluded that the level of performance is consistent with the professional profile, showing that they are competent in any setting in direct relation to personal, professional and social references.

Keywords: University training; graduates; employers; professional skills 


\section{INTRODUCCIÓN}

En Colombia, el ejercicio profesional en egresados de Instituciones de Educación Superior (IES), refleja la calidad de los procesos académicos recibidos durante la etapa formativa (MEN, 2017). En la década de los ochenta se difundió un concepto, como propósito de educación, definido como competencia, el cual permitió la delimitación y unificación de Educación Basada en Competencias. Esta meta educativa encuentra soporte en los lineamientos del Proyecto Tuning a nivel mundial y Alfa Tuning para América Latina, donde se concluye que la educación de alta calidad se encuentra sujeta al cumplimiento de los estándares en términos de competencias profesionales, competencias en términos de los créditos exigidos, mecanismos e instrumentos de evaluación, procesos de acreditación de las instituciones y procesos de formación para la investigación y la innovación (Irigoyen, Jiménez y Acuña, 2011).

De esta manera se espera que la educación basada en competencias supere las diferencias entre el saber y el saber hacer, fundamentados en los perfiles de egreso, para viabilizar la participación de los profesionales egresados en un mundo laboral altamente competente. En correspondencia con el Proyecto Alfa Tuning, el Ministerio de Educación Nacional-MEN publica en 2003 el Decreto 2566, "por el cual se establecen las condiciones mínimas de calidad y demás requisitos para el ofrecimiento y desarrollo de programas académicos de educación superior y se dictan otras disposiciones" (Decreto 2566, 2003). Los lineamientos de este decreto, permiten reconocer que las competencias son conocimientos, prácticas, experiencias, habilidades y destrezas que desarrolla un individuo para percibir, comprender, convertir, evolucionar, transformar, informar, prevenir y participar en un universo cambiante (Lemus, 2016). Esta noción integra: el saber, saber hacer, saber ser y el saber convivir (Rodríguez, 2007).

De esta manera una competencia no es solo el conocimiento, sino también la habilidad para utilizar ese conocimiento en una determinada situación, en otros términos, poder transcender del aprendizaje dentro del aula de clase a la solución de problemas en los diferentes contextos, según Cruz y Barrios (2010) "la evaluación por competencias favorece la objetividad, unifica criterios y facilita la especificación de las debilidades con fines de mejoramiento" (p. 4).

$\mathrm{Al}$ respecto se puede considerar que las competencias institucionales y la formación del individuo, para la formación académica-profesional y para la capacitación en el ejercicio profesional, se centran en la calidad humana, adecuada formación profesional y en la preparación eficiente para una vida personal y laboral. En los empleadores la preparación evidenciada en los profesionales se corresponde con un desempeño acorde a las necesidades del contexto laboral de acuerdo a la disciplina, lo anterior contrastado con el perfil profesional planteado por las instituciones educativas y programas académicos en coherencia con el sector educativo, laboral y competitivo.

En este contexto, la Universidad de Boyacá, Colombia, desde su Misión y Visión ha definido al egresado como "Profesional formado con libertad, criticidad y compromiso social, lo cual se evidencia en la calidad académica y humana de su ejercicio profesional desde cada disciplina” (Universidad de Boyacá, 2017b, p. 15). 
La Institución concibe el perfil de egreso como un sistema dinámico de interacción, fundamentado en la formación integral donde se encajan cada uno de los elementos del proceso de formación que involucra el desarrollo de competencias, saberes, valores, elementos disciplinares, investigativos, tecnológicos y de proyección social. Esta interacción permitirá la transformación del aspirante en un profesional altamente competente (Universidad de Boyacá, 2013, p. 6).

En el contexto nacional e internacional, Libera (2007) y Ferro (2014) han presentado algunas metodologías aplicadas en diversas IES, referentes a investigaciones con base en seguimiento y opinión de empleadores de acuerdo a trayectoria y desempeño profesional, las cuales son pertinentes para la construcción de un modelo sólido y adecuado evaluando las competencias con base en culturas, preparaciones, prácticas, experiencias, conductas y procedimientos, permitiendo conocer la percepción de contratantes frente al ejercicio de los profesionales (Libera, 2007).

El estudio es desglosado en referencia a la percepción de los profesionales egresados de la Universidad de Boyacá, de esta forma se consideró evaluar competencias, formación y desempeño profesional; la percepción de los empleadores respecto a estas categorías, constituye el aspecto central del presente análisis.

\section{Referentes Conceptuales}

De acuerdo con la literatura, la definición competencia viene siendo utilizada desde el siglo XVI; no obstante, en 1960 fue incluida en el área laboral, caracterizando a los trabajadores con adecuados desempeños; seguido a ello, se inmerso a los saberes del lenguaje. Actualmente, es referido como Enfoque Basado en Competencias (EBC), algunos referentes teóricos coinciden, en que es un término novedoso y amplio, teniendo en cuenta, que varios postulados lo caracterizan y orientan a varias reestructuraciones educativas en diversos países a finales del siglo XX: sustentando, que lo novedoso del término, es que se enfatiza en la articulación, interdisciplinaridad y relación de diferentes áreas del conocimiento según lo referido por Ruiz, Jaraba y Romero (2005), Coll (2007) y Díaz (2006) en sus postulados, quienes enfocados al término, llegan a un concepto, caracterizado por compendios, en el ámbito educativo, con enfoque pedagógico, situando su aplicación en el esquema curricular o contenido programático.

El profesional de la Universidad de Boyacá (Colombia) es un individuo que en su transcurrir académico incorporó conocimientos, competencias, destrezas, prácticas, experiencias, valores y cualidades evidenciadas en el ejercicio profesional; acorde a estas particularidades, se pude inferir que el desempeño profesional corresponde con la formación recibida, enfatizando que las fortalezas, conductas, actuaciones, consecuencias o derivaciones resultantes de su desempeño, evalúan el ejercicio profesional; de otra parte, garantiza su ética, liderazgo, integralidad, compromiso social, afrontamiento a la incertidumbre y los retos sociales; principios consagrados en la misión y en el modelo pedagógico institucional (Universidad de Boyacá, 2013a); de esta manera, se espera que la formación basada en competencias supere las diferencias entre el ser, el saber hacer y el saber conocer lo que propone entre otras condiciones, desarrollar perfiles profesionales en términos de competencias, que le permita al egresado la participación exitosa en un mundo laboral altamente competente. 
En el ámbito de la educación poco se ha estudiado sobre la relación que tiene el enfoque académico frente al desempeño profesional; los estudios realizados a los empleadores representan una herramienta de interés para las Universidades e IES, logrando conocer elementos fundamentales que aportarán significativamente a la apreciación, valoración, eficacia, congruencia y formación académica impartida en la institución.

De acuerdo con Ferro (2014) una de las funciones de las Universidades es establecer una responsabilidad social frente a las necesidades de impartir conocimientos a los estudiantes de una manera tal, que desarrollen en la sociedad un papel de cambio, de transformaciones de las condiciones de vida actuales hacia planos más justos, más humanos y cualitativos. Al respecto, es importante que las IES de manera constante, actualicen sus contenidos programáticos en función de cambios que a nivel social se presentan, buscando de esta manera que el egresado adquiera las herramientas tanto profesionales como humanas que le permitan impactar de manera positiva en la sociedad. Estas herramientas se resumen en las competencias generales y específicas, que asociadas a la productividad y la competitividad (MEN, 2020a), se fundamentan en función de entregar a los profesionales oportunos y congruentes conceptos, metodologías que le faciliten desempañarse de manera adecuada en su ejercicio profesional, lo que va unido a que la educación prepare al profesional para afrontar con rectitud y responsabilidad los inconvenientes, adversidades y frustraciones propias en su ejercicio profesional y para quienes los contratan (Arévalo, Morales y Meneses, 2012).

Un enfoque integrado del concepto de competencia laboral permite a las IES evaluar la pertinencia de sus currículos en virtud de su construcción, ofreciendo a las instituciones un índice de calidad. Ante este panorama y en búsqueda de obtener datos objetivos para dar respuesta a las condiciones planteadas, las IES realizan investigaciones permitiendo visualizar con claridad la realidad a la que los profesionales egresados se enfrentan, enfocadas en sus fortalezas y debilidades, para de esta manera poder ajustar los contenidos académicos en los programas formativos a las condiciones y necesidades del entorno social, empresarial y académico, procurando así generar calidad en la formación de profesionales competentes y ajustados al contexto (Universidad del Magdalena, 2014).

Para Castillo, Ohao y Schleser (2014), las trayectorias laborales de los egresados permiten medir, analizar y ajustar la formación impartida acorde con el desempeño. En su trabajo caracteriza y evalúa los avances laborales de los egresados, mostrando las formas como estos determinan procesos enfocados al nivel diferenciado alcanzado y en la estructura de la empresa, trayectorias estacionarias de acuerdo al sector económico de empleabilidad, avances positivos según la contratación y el área de desempeño o función realizada con la formación complementaria y trayectos derogados según la remuneración salarial obtenida.

Con esto se busca analizar las falencias y fortalezas del ejercicio académico realizado al interior de las IES. Aspecto fundamental de las universidades e IES que aparece como indicador cuantitativo de validez y producto es la calidad, el cual se enlaza con las particularidades, consideradas cualitativas, de los métodos, técnicas, conocimientos y metodologías universitarias. El desempeño profesional inquieta no solo a los participantes en el proceso educativo (directivos, docentes y estudiantes); sino también a los contratantes y empleadores que consideran a las universidades o IES cono escenarios de aprendizaje de profesionales y de producción de conocimiento fundamentales para conservar el desempeño profesional y laboral. 
En la actualidad, el estudiante se considera el eje central en el proceso de aprendizaje formativo, atrás se ha dejado la concepción de que el profesional es aquel que cuenta con la preparación, destrezas, prácticas, experiencias, entre otras características para desempeñarse con éxito en una profesión específica (González y González, 2008). Actualmente, las competencias son necesarias para ejecutar o desempeñar un función específica, sin tener en cuenta, el entorno, obligación, compromiso, sector o actividad (MEN, 2020b); además, están relacionadas con la formación universitaria (Muñoz, Rodríguez, Hincapié, Agudelo y Ramírez, 2012).

Mediante este tipo de estudios, se orienta a las Universidades e IES a establecer el seguimiento de los profesionales, identificando aspectos fundamentales que orienten a un plan de mejora en el proceso formativo donde se integre al empleador, quien, desde su percepción en el ámbito cultural, económico y social permita evidenciar el impacto profesional generado en el medio y contexto laboral.

\section{MetodoloGía}

Estudio bajo enfoque cuantitativo de tipo transversal descriptivo y fase analítica. Se tomó como población de referencia a las empresas que tienen vinculados profesionales, equivalente a 862 egresados de la Universidad de Boyacá. Se seleccionó una muestra aleatoria con una confianza del 95\%, precisión del 5\% y una proporción esperada del 50\%, para una cobertura de 188 empleadores. Se identificaron a los jefes directos quienes fueron abordados de manera personal, telefónicamente o vía correo electrónico, por los coordinadores de tutoría de egresados de la institución y aceptaron participar voluntariamente en el estudio. Acorde a estas tipologías los empleadores entrevistados corresponden a organizaciones de orden público o privado, algunas de ellas forman parte o han participado como sedes de prácticas formativas, conociendo el proyecto educativo institucional, y contribuyendo en la formación como tutores, coordinadores o docentes de práctica.

A través de escala evaluativa, las variables estudiadas fueron los conocimientos y valores, con referencia a las competencias generales se evaluaron las brechas y se plantean diferentes reactivo desde el fundamento de las características y atributos señalados en el perfil de egreso de la Universidad de Boyacá, de acuerdo a la escala de respuesta de 1 a 3 equivalente a aceptable, alto y muy alto, respectivamente; se calificó formación y desempeño; con referencia a las brechas se evalúo la importancia y logro de las competencias. Esta escala en su versión final quedó integrada por 5 apartados incluyendo 40 ítems, previamente se sometió a la prueba piloto por 5 empleadores externos, con el objetivo de fin de establecer la estructuración y claridad del instrumento.

Entre enero 2018-2019, se aplicaron las encuestas a los empleadores de las organizaciones participantes en el estudio. El análisis de los resultados se realizó por medio del paquete estadístico SPS ${ }^{\circledR}$, versión 25.0; los datos obtenidos para variables categóricas se formularon en frecuencias absolutas y relativas; para las variables numéricas medidas de tendencia central, finalmente, la asociación por medio de chi-cuadrado.

Los aspectos éticos se sustentan bajo la firma de consentimiento informado, privacidad de información de empresas y empleadores; contando con aval institucional por parte del comité de bioética. El aspecto financiero fue cubierto por la Universidad de Boyacá. 


\section{Resultados y Discusión}

Al efectuar la evaluación de las características de la empresa, se evidenció que el 78.2\% de los egresados se encuentran ubicados laboralmente en empresas locales y el 19.7\% en nacionales, un porcentaje menor se ubican en empresas internacionales. Como se muestra en la Tabla 1, el alcance de la empresa está definido a nivel nacional, otorgando un 89\% [IC 95\% 84.2-93.7], siendo el principal foco de estudio, seguida de la empresa multinacional con presencia en Colombia con el 5\% [IC 95\% 1.0-18.0], dichos resultados podrían justificarse por el consultor de Deloitte, quien manifestó que: "Colombia cuenta con el 6\% de las multiláteras de la región" (Lozano, 2016, párr. 11).

La constitución legal de las diferentes empresas, evidencian que el 93\% [IC 95\% 89.1-96.8] son de personería jurídica, el restante de ellas denota estar compuesta por una persona natural o física. Asimismo, se evidenció que el 50\% [IC 95\% 39.9-60.0] son de naturaleza privada, seguida de las públicas con un 47\% [IC 36.5-57.4], los demás indican pertenecer a empresas mixtas, ONG u otra, datos que difieren con Cruz y Barrios (2010), quienes

TABLA 1.

Características de las empresas objeto de estudio

\begin{tabular}{|c|c|c|c|c|}
\hline Variable & $\mathrm{n}$ & $\%$ & \multicolumn{2}{|c|}{ Intervalo de Confianza 95\% } \\
\hline Alcance de la empresa & & & Límite Inferior & Límite Superior \\
\hline Empresa multinacional con presencia en Colombia & 10 & $5 \%$ & 1.0 & 18.0 \\
\hline Empresa colombiana presencia en el exterior & 6 & $3 \%$ & 1.0 & 16.6 \\
\hline Empresa Nacional & 164 & $89 \%$ & 84.2 & 93.7 \\
\hline Empresa Internacional & 5 & $3 \%$ & 1.0 & 16.6 \\
\hline \multicolumn{5}{|l|}{ Naturaleza de la empresa } \\
\hline Pública & 88 & $47 \%$ & 36.5 & 57.4 \\
\hline Privada & 95 & $50 \%$ & 39.9 & 60.0 \\
\hline Mixta & 1 & $1 \%$ & 1.0 & 14.0 \\
\hline $\mathrm{ONG}$ & 2 & $1 \%$ & 1.0 & 14.0 \\
\hline Otra & 2 & $1 \%$ & 1.0 & 14.0 \\
\hline \multicolumn{5}{|l|}{ Constitución legal } \\
\hline Persona Natural o Física & 12 & $7 \%$ & 1.0 & 21.4 \\
\hline Persona Jurídica & 165 & $93 \%$ & 89.1 & 96.8 \\
\hline \multicolumn{5}{|l|}{ Número de empleados } \\
\hline Menos de 11 Empleados & 25 & $13 \%$ & 1.0 & 26.1 \\
\hline Entre 11 y 50 Empleados & 39 & $21 \%$ & 8.0 & 33.7 \\
\hline Entre 51 y 200 Empleados & 35 & $19 \%$ & 6.0 & 31.9 \\
\hline Más de 200 Empleados & 89 & $47 \%$ & 36.5 & 57.4 \\
\hline
\end{tabular}

Fuente: Autores. 
refiere en su estudio, que el 90\% de los encuestados se encuentra vinculados en el sector público; discrepando estos resultados se puede inferir que los egresados de la Universidad de Boyacá (Colombia) se centran en las instituciones privadas debido a la baja posibilidad de postulación en instituciones públicas, así como, lograr aplicar a las convocatorias que estas entidades generan; por otro lado, se podría incidir circunstancias salariales, componentes políticos, entre otras características que influyen en la vinculación a este tipo de organizaciones.

Bajo criterio organizativo, referente a las micro pequeña y mediana empresa, en Colombia (Ley 905, 2004), se define el tamaño según el número de empleados y los activos totales con los que cuenta. En términos de planta de personal, una micro, pequeña, mediana y gran empresa poseen entre 1 y 10, 11 y 50, 51 y 200; y más de 200 empleados respectivamente. De acuerdo a lo anterior, a nivel general el 47\% [IC 36.6-57.4] de los empleadores encuestados en el presente estudio, pertenecen a grandes empresas (Tabla 1).

\section{Competencias genéricas}

Como proyecto estratégico, la Universidad de Boyacá (Colombia) ha incorporado en su proceso académico la formación basada en competencias, con el objetivo de beneficiar el esquema integrador del educando bajo un plan flexible e interdisciplinar centrado y enfocado en la misión y visión institucional (Universidad de Boyacá, 2013b).

El análisis de competencias genéricas, al momento de su evaluación por brechas, consideró la importancia que da el empleador a cada elemento y el grado de cumplimiento que observa en el desempeño del egresado. Lo anterior, para determinar la brecha entre ambas calificaciones y así identificar las competencias consideradas fortalezas o debilidades en el Perfil de Egreso de la Universidad de Boyacá (Figura 1).

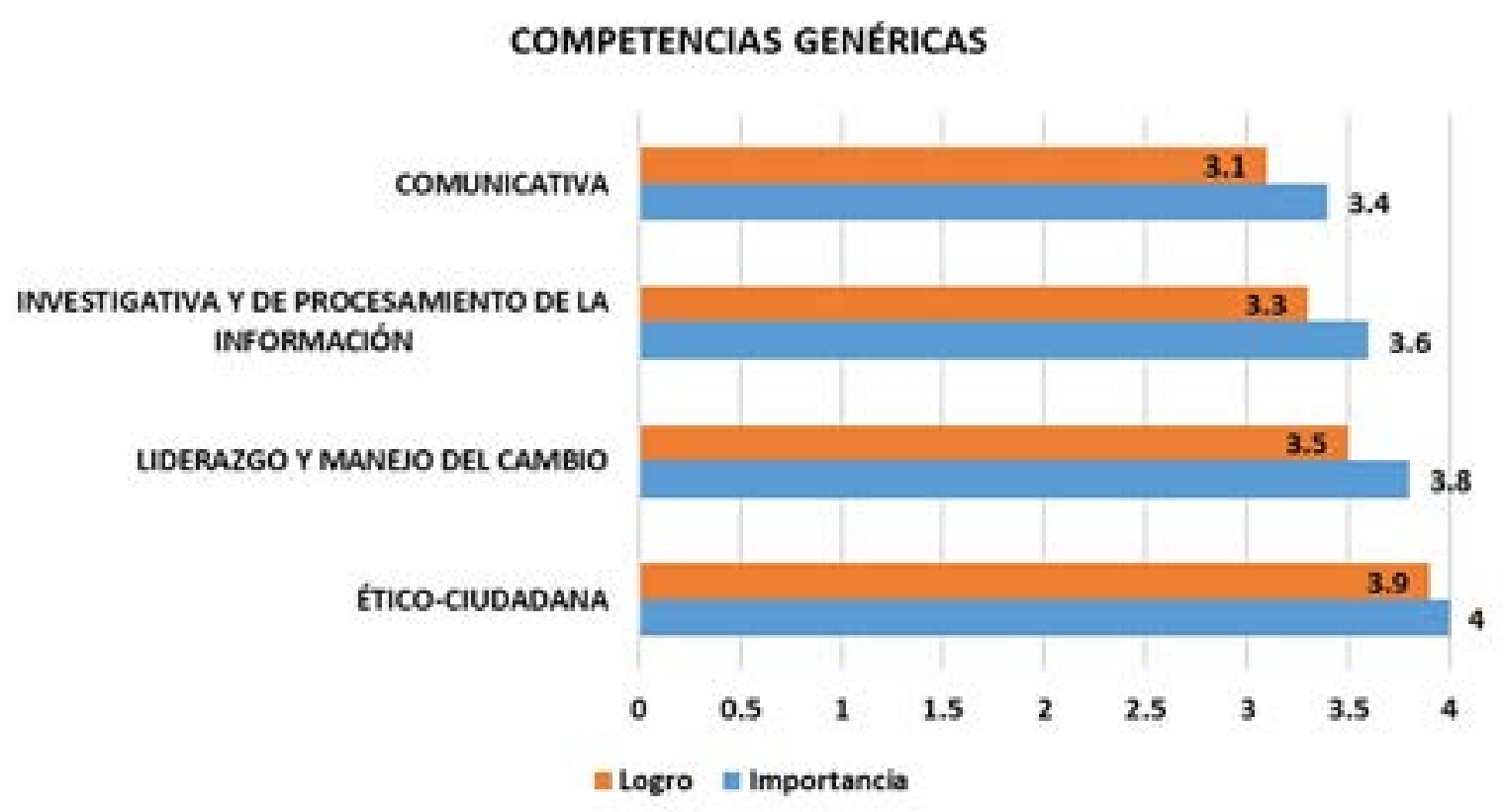

Figura 1. Brecha importancia/ logro competencias genéricas Universidad de Boyacá. (0-Mínimo, 4-Máximo).

Fuente: Autores. 
En el análisis de las competencias institucionales, la brecha promedio más alta se observa en la competencia comunicativa donde se resalta principalmente la comunicación de pensamientos, ideas e información por escrito; lo anterior nos permite inferir que los egresados de la Universidad de Boyacá son capaces de poner en práctica habilidades comunicacionales asertivas y continuadas, y, por lo tanto, de actitudes afectivas dentro de los procesos, a través de un lenguaje escrito y oral.

Con respecto a la competencia investigativa y de procesamiento de la información (Universidad de Boyacá, 2013), se observó que está orientada a gestionar conocimientos para la comprensión y solución de las necesidades, que procuren el mejoramiento de proceso de investigación que susciten la solución de problemas aplicando el método científico y asumiendo un pensamiento crítico para resolver imponderables, por lo que el egresado, es un profesional que demuestra tener la capacidad de utilizar herramientas informáticas, tener la disposición de aprender y mantenerse actualizado, teniendo en cuenta la opinión de los empleadores.

La brecha que representa la competencia de liderazgo y manejo del cambio resalta la capacidad del egresado de adaptarse fácilmente a los cambios y resolver fácilmente imponderables. Finalmente, en menor porcentaje de brecha se presentó la competencia ético-ciudadana, evidenciando que el egresado tiene excelentes competencias ciudadanas y que los empleadores reconocen en ellos la formación en valores y principios éticos.

Los empleadores valoran, principalmente, las competencias generales; en este sentido infiere con lo expresado por Freire, Teijeiro y Pais (2011), evidenciando similitud en la evaluación de competencias generales, siendo estas las más valoradas y ratificadas en el mercado laboral; aspecto igualmente resaltado por Palmer, Montaño y Palou (2009), Muñoz et al. (2012) y Jackson (2012) en sus estudios.

Evaluar el desempeño de los profesionales, por medio de los empleadores, expresa un nivel paralelo a las competencias generales; así como, en los valores institucionales, no obstante, es fundamental identificar aspectos de mejora en orden a la organización o empresa, con base al cliente interno y externo, perfeccionamiento del servicio, intercambio de ideas, estrategias y actividades que fomenten la transformación, negociación en pro del trabajo colaborativo, relaciones interpersonales, convivencia pacífica, destreza y afrontamiento al cambio; igualmente motivando a fortalecer la capacidad crítica y liderazgo. En concordancia con esto y de acuerdo a lo evidenciado en los resultados presentados por la Universidad de Valencia (2007), narró que se destacaba entre la población estudiada, características en las competencias generales centradas en, resolución de problemas, responsabilidad, trabajo en equipo, planificación, planeación y gestión de recursos, según lo referido por Peiro, Roig, González-Roma y Gamboa (2009).

\section{Niveles de satisfacción con respecto a la formación del egresado}

La Figura 2, evidencia que el nivel de satisfacción entre formación y necesidades de la empresa se ubican en un nivel alto correspondiente al 67, 91\% [IC 95\% 59-72], seguido de un nivel muy alto con un 21,93\% [IC 95\% 16-27], finalmente un 10,16\% [IC 95\% 5-14] de los empleadores refieren un nivel aceptable con respecto a la formación de los egresados. 


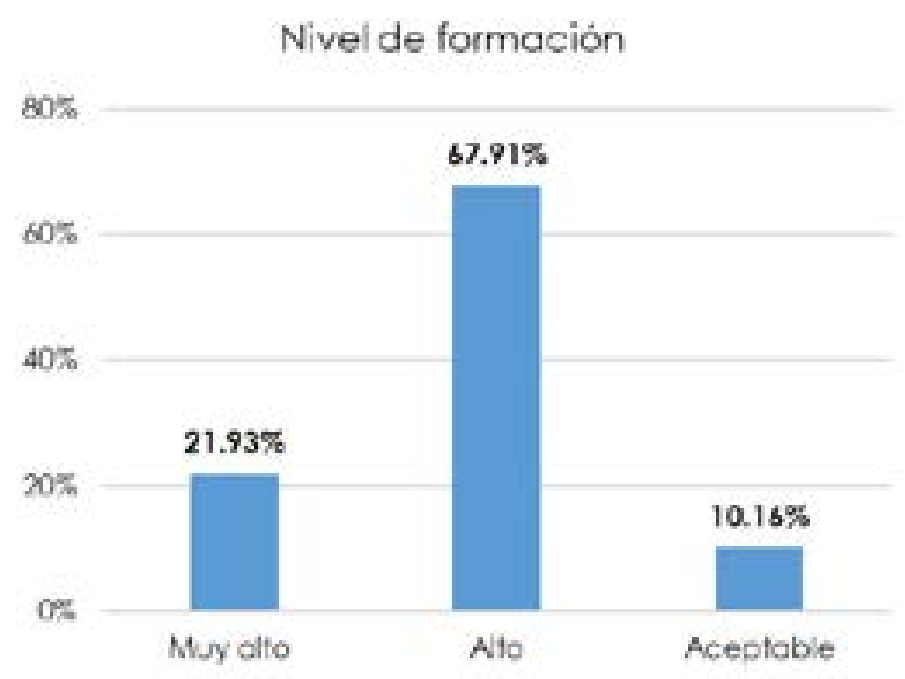

Figura 2. Nivel de formación de los egresados de la Universidad de Boyacá según opinión de empleadores.

Fuente: autores.

\section{Niveles de satisfacción con respecto al desempeño del egresado}

La Figura 3, evidencia la satisfacción que tiene el empleador con respecto al desempeño laboral del egresado, el 65,78 [IC 95\% 59-72] lo evalúan en un nivel alto, seguido de un 25,67\% [IC 95\% 19-32] quienes consideran un nivel muy alto; finalmente, el 8,56\% [IC 95\% 4-11] considera que el desempeño laboral de los profesionales es aceptable; lo que significa en los egresados concordancia entre las competencias y los requerimientos exigidos por las empresas contratantes.

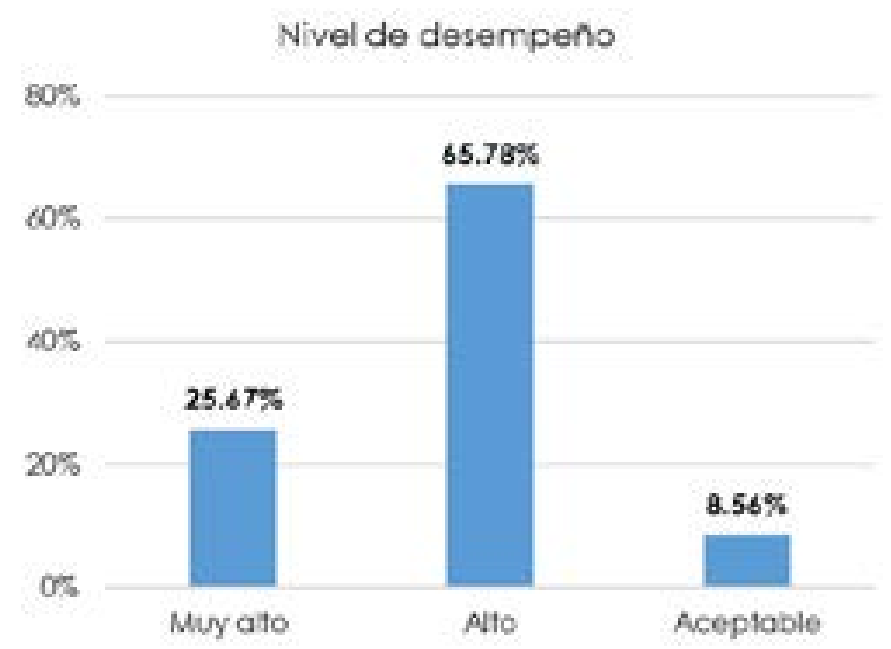

Figura 3. Nivel desempeño de egresados UdB según opinión empleadores.

Fuente: autores

La información obtenida sobre el nivel de formación deja claro que se están preparando profesionales íntegros, con capacidad de análisis y acorde a las necesidades del contexto; asimismo, en sus actuaciones los profesionales formados son capaces de enfrentar la incertidumbre. 
El proyecto Tuning en América Latina (Beneitone, Esquenetini, González, Maletá, Siufi y Wagenaar, R. 2007), refiere resultados diferentes, considerando que el liderazgo y trabajo en equipo, no son aspectos contemplados en la evaluación referida por los empleadores; de otro lado, el estudio desarrollado por Cabeza, Castrillón y Lombana (2017) arrojó datos que evidencian que la capacidad para identificar, proyectar, concebir, solventar, solucionar o resolver problemas; así como, toma de decisiones y compromiso ético, son los aspectos más relevantes; datos similares a los encontrados por Cabrera, López y Portillo (2016) quienes resaltaron la relevancia en el trabajo en equipo y liderazgo competencias denominadas interpersonales y sistémicas.

Respecto al nivel de desempeño, el 65,7\% de los empleadores consideran que los egresados tienen una buena formación, lo cual ha sido inculcado durante su formación académica, asimismo, refieren comunicación asertiva, experiencia y práctica en las labores desempeñadas, son comprometidos, responsables, organizados proyectando adecuadamente sus actividades; además, demuestran gusto y agrado con la labro desempeñada, datos similares arrojados por Jaramillo, Montesino, Suárez y Vergara (2017), donde el 84\% de los empleadores coinciden con las apreciaciones anteriormente referidas, todo esto basado en el perfil de egreso de la Universidad de Boyacá, el cual plantea formular egresados libres, críticos y comprometidos socialmente, reflejado en el ejercicio profesional y evidenciado en la calidad humana (Universidad de Boyacá, 2017), expresado en su mayoría de forma sobresaliente para la institución y el empleador.

La responsabilidad, trabajo en equipo, liderazgo, ética y comunicación son las fortalezas más desatacadas en los profesionales de la Universidad de Boyacá, Colombia, desde la perspectiva de los empleadores, esto gracias a la ejecución del Modelo Pedagógico Institucional, el cual se centra en la formación basada en competencias.

Por lo anterior es importante tener en cuenta, que la formación por competencias, demarca las competencias profesionales, las cuales deben ser claras con el fin de ser reflejadas en el desempeño profesional. Villa y Poblete (2007) refirieron que estas características compensan metas personales y profesionales, en pro de un proyecto ético de vida; datos afines a los de Muñoz et al. (2012), quien considera a las competencias analizadas como fortalezas, datos análogos a los reportados por Peterson y Sánchez (2016) refiriendo "los egresados desde la perspectiva del empleador están capacitados en habilidades y competencias básicas para su desempeño profesional, específicamente en responsabilidad social y ética, disciplina, organización y trabajo en equipo" (p. 235), ratificando que los profesionales deben integrar competencias que resalten su ejercicio profesional acorde a la disciplina e contexto donde se desempeñan.

\section{Relación nivel de formación y nivel de desempeño}

De acuerdo a los resultados que muestra la Tabla 2, existe relación estadísticamente significativa entre formación y desempeño. De otra parte, los resultados indican que los empleadores, en mayor proporción (65,8\%), están altamente satisfechos, con la formación, el ejercicio profesional y laboral de los profesionales graduados de la Universidad de Boyacá en las diferentes disciplinas. 
TABLA 2.

Relación estadística entre formación y desempeño de los egresados

\begin{tabular}{|c|c|c|c|c|c|}
\hline \multirow{2}{*}{$\frac{\text { Relación }}{\text { Nivel de Formación }}$} & \multicolumn{3}{|c|}{ Nivel de Desempeño } & \multirow{2}{*}{$\mathrm{X} 2$} & \multirow{2}{*}{$\mathrm{p}$} \\
\hline & Muy alto & Alto & Aceptable & & \\
\hline Muy alto & $18.2 \%$ & $3.2 \%$ & $0.5 \%$ & \multirow{4}{*}{141.01} & \multirow{4}{*}{0.00} \\
\hline Alto & $7.0 \%$ & $58.3 \%$ & $2.7 \%$ & & \\
\hline Aceptable & $0.5 \%$ & $4.3 \%$ & $5.3 \%$ & & \\
\hline Total & $25.7 \%$ & $65.8 \%$ & $8.5 \%$ & & \\
\hline
\end{tabular}

Fuente: Autores.

\section{Conclusiones}

A partir de la perspectiva de empleadores, se evidencia que los profesionales están formados en competencias para desempeñarse laboralmente resaltando la comunicación, investigación, procesamiento de la información, liderazgo y manejo del cambio, permitiendo percibir la labor desarrollada por la Institución académica fundamentada en la formación integral, con el objetivo de las necesidades del medio laboral con un desempeño acorde a las exigencias de la disciplina.

Se infieren debilidades en aspectos, que pueden afectar el desarrollo de las actividades, pero que para la institución se deben tomar como acciones de mejora en la implementación y reestructuración curricular.

Se evidencia un grado de satisfacción entre alto y muy alto en la formación y desempeño de los egresados, resaltando la valoración que hacen los empleadores de la habilidad de observación, investigación, síntesis, liderazgo y trabajo colaborativo; asimismo, fueron bien valoradas por lo empleadores las competencia comunicativa donde se resalta principalmente la comunicación de pensamientos, ideas e información por escrito; infiriendo que los egresados de la Universidad de Boyacá son capaces de poner en práctica habilidades comunicacionales asertivas y continuadas, y, por lo tanto, de actitudes afectivas dentro de los procesos, a través de un lenguaje escrito y oral.

Finalmente, los resultados, señalan que la incorporación de competencias genéricas en la gestión académica corresponde a las necesidades del sector productivo, partiendo del análisis de la brecha entre la importancia que tiene para los empleadores la capacidad de manejar los contextos propios de la formación del profesional y el logro del egresado de la Universidad de Boyacá en el contexto laboral.

\section{REFERENCIAS}

Arévalo, G., Morales B. y Meneses, L. D. (2012). Exploración cualitativa y cuantitativa de la realidad del egresado del programa de Administración de Empresas de la Universidad de La Salle. Año 2008 primera parte. [Trabajo de grado]. Unisalle, Bogotá, D.C., Colombia. Disponible en https://ciencia.lasalle.edu.co/administracion_de_empresas/1056

Beneitone, P., Esquenetini, C., González, J., Maletá, M., Siufi, G. y Wagenaar, R. (eds.) (2007). Reflexiones y perspectivas de la educación superior en América Latina. [Informe final Proyecto Tuning América Latina: 2004-2007]. Groninga y Bilbao: Universidad de Deusto y Universidad de Groningen. Recuperado de http://tuningacademy.org/wp-content/ uploads/2014/02/TuningLAIII_Final-Report_SP.pdf 
Cabeza, L. Castrillón, J. y Lombana, J. (2107). Importancia y coincidencia de competencias para egresados de administración y empleadores: un enfoque por regiones de Colombia. Revista Facultad de Ciencias Económicas, 25(2), 105-122. http://dx.doi.org/10.18359/ rfce.1983

Cabrera, N., López, L. y Portillo, M. (2016). Las competencias de los graduados y su evaluación desde la perspectiva de los empleadores. Estudios Pedagógicos, 42(3), 69-87. http:// dx.doi.org/10.4067/S0718-07052016000400004

Castillo, V., Ohao, M. y Schleser, D. (2014). Evaluación de impacto en la inserción laboral de los beneficiarios de los cursos sectoriales de formación profesional. Serie Documentos de Trabajo. Buenos Aires: OIT. Disponible en http://www.ilo.org/public/libdoc/ ilo/2014/486341.pdf

Coll, C. (2007). Las competencias en la educación escolar: algo más que una moda y mucho menos que un remedio. Revista Aula de Innovación Educativa, (161), 34-39. Disponible en https://www.grao.com/es/producto/las-competencias-en-la-educacion-escolar-algomas-que-una-moda-y-mucho-menos-que-un-remedio

Cruz, N. y Barrios, M. (2010). Opinión de Empleadores sobre desempeño de egresados de la Facultad de Enfermería, Poza Rica, México. ene. Revista de Enfermería, 4(2), 40-44. Disponible en http://ene-enfermeria.org/ojs/index.php/ENE/article/view/131

Díaz, B. (2006). El enfoque de competencias en la educación. ¿Una alternativa o un disfraz de cambio? Perfiles Educativos, 28(111), 7-36. Disponible en http://www.iisue.unam.mx/ perfiles/numeros/2006/111

Ferro, J. (2014). Visión de la Universidad ante el siglo XXI. [2 ed.]. Barranquilla: Editorial Universidad del Norte. Disponible en https://www.uninorte.edu.co/ documents/3055771/4f34a62b-2651-433b-867a-62c8a1d772f5

Freire, M., Teijeiro, M. y Pais, C. (2011). Políticas educativas y empleabilidad: ¿cuáles son las competencias más influyentes? Archivos Analíticos de Políticas Educativas, (19), 1-24. Disponible en https://epaa.asu.edu/ojs/article/view/911

González, M. y González, T. (2008). Competencias genéricas y formación profesional: Un análisis desde la docencia universitaria. Revista Iberoamericana de Educación, (47), 185-209. Recuperado de https://rieoei.org/historico/documentos/rie47a09.pdf

Irigoyen, J., Jiménez, M. y Acuña, K. (2011). Competencias y Educación Superior. Revista Mexicana de Investigación Educativa, 16(48), 243-266. Disponible en https://www.comie.org.mx/revista/v2018/rmie/index.php/nrmie/article/view/367

Jackson, D. (2012). Business undergraduates' perceptions of their capabilities in employability skills: Implications for industry and higher education. Industry and Higher Education, 26(5), 345-356. https://doi.org/10.5367/ihe.2012.0117

Jaramillo, Q., Montesino, A., Suárez, T. y Vergara, A. (2017). Opinión de empleadores sobre las competencias de los egresados del programa de Enfermería de la Universidad de Sucre frente a los retos cotidianos que enfrentan en el desempeño de sus funciones. [Tesis de grado]. Universidad de Sucre, Sincelejo, Colombia. Disponible en: https://repositorio.unisucre.edu.co/bitstream/001/636/1/T610.732\%200\%2061.pdf

Lemus, L. (agosto 23, 2016). Competencias laborales: ¿Qué son y para qué sirven? [Online]. Disponible en https://sueldosysalarios.com.mx/que-son-las-competencias-laborales-y-para-que-sirven/

Libera, B. (2007). Impacto, impacto social y evaluación del impacto. ACIMED, 15(3). Disponible en http://www.ecimed.sld.cu/ 
Lozano, R. (agosto 5, 2016). Empresas del país renuevan su interés de intervenir por fuera. El Tiempo. Disponible en http://www.eltiempo.com/economia/empresas/empresas-colombianas-con-inversiones-en-el-extranjero-33529

Muñoz, A., Rodríguez, F., Hincapié, C., Agudelo, G. y Ramírez, C. (2012). Percepción de empleadores sobre las competencias de graduados del programa de Enfermería de la Fundación Universitaria del Área Andina de Pereira (Colombia). Revista Cultura del Cuidado, (9), 22-38. Disponible en: http://repositorio.unilibrepereira.edu.co:8080/perei$\mathrm{ra} / \mathrm{handle} / 123456789 / 168$

Palmer, P., Montaño, M. y Palou, M. (2009). Las competencias genéricas en la educación superior. Estudio comparativo entre la opinión de empleadores y académicos. Revista Psicothema, 21(3), 433-438. Disponible en: http://www.psicothema.com/pdf/3650.pdf

Peiró, J., Roig, J., González-Roma, R. y Gamboa, J. (2009). Estudio de las demandas de los empleadores de titulados universitarios de la Provincia de valencia. [Síntesis de resultados]. Valencia: Observatorio de Inserción Profesional y Asesoramiento Laboral-OPAL y Universidad de Valencia. Disponible: https:/www.uv.es/opalweb/estudi\%20i\%20analisi/ Analisi\%20demanda\%20empresari/Primer\%20estudi/Sintesi_de_Informe_General_ de_Resultats.pdf

Peterson, J. y Sánchez, D. (2016). Percepción del empleador: competencias del egresado de Enfermería de la Universidad Popular del Cesar. Advocatus, 13(26), 227-237. http:// dx.doi.org/10.18041/0124-0102/advocatus.26.944

República de Colombia. Congreso de la República. (agosto 2 de 2004). Por medio de la cual se modifica la Ley 590 de 2000 sobre promoción del desarrollo de la micro, pequeña y mediana empresa colombiana y se dictan otras disposiciones. [Ley 905]. Diario Oficial No. 45.628. Disponible en http://www.comunidadcontable.com/BancoMedios/Documentos\%20PDF/ley_905_de_2004.pdf

República de Colombia. MEN. (2020a). Competencias laborales generales. [Online]. Disponible en http://www.mineducacion.gov.co/1621/article-79387.html

República de Colombia. MEN. (2020b). Instituciones de Educación Superior. Clasificación de las Instituciones de educación Superior (IES). [Online]. Disponible en https://www.mineducacion.gov.co/portal/Educacion-superior/Sistema-de-EducacionSuperior/231240:Instituciones-de-Educacion-Superior

República de Colombia. MEN. (10 de septiembre de 2003). Por el cual se establecen las condiciones mínimas de calidad y demás requisitos para el ofrecimiento y desarrollo de programas académicos de educación superior y se dictan otras disposiciones. [Decreto 2566]. DO: 445.308. Disponible en https://www.mineducacion.gov.co/portal/

República de Colombia. MEN. (2017). Articulación de la Educación con el Mundo Productivo. Competencias Laborales Generales. [Online]. Disponible en https://www.mineducacion. gov.co/1621/articles-106706_archivo_pdf.pdf

Rodríguez, Z. (2007). El paradigma de las competencias hacia la educación superior. Revista Facultad de Ciencias Económicas, de la Universidad Militar Nueva Granada. 15(1), 145-165. Disponible en https://revistas.unimilitar.edu.co/index.php/rfce/article/ view/4554

Ruiz, M., Jaraba, B. y Romero, S. (2005). Competencias laborales y la formación universitaria. Revista Psicología desde el Caribe, (16), 64-91. Disponible en http://rcientificas. uninorte.edu.co/index.php/psicologia/article/view/1996

Universidad de Boyacá. Rectoría. (septiembre 26 de 2017a). Perfil de Egresado Universidad de Boyacá. [Resolución $N^{\circ} 188$ ]. Disponible en https://www.uniboyaca.edu.co/es/node/4335 
Universidad de Boyacá. Rectoría. (diciembre 15 de 2017b). Competencias generales de la institución y específicas de los programas. [Acuerdo No. 1048 A]. Disponible en https:// www.uniboyaca.edu.co/es/centro-informacion/documentos

Universidad de Boyacá. Rectoría. (marzo 21 de 2013a). Proyecto Educativo Institucional. [Acuerdo No. 066]. Disponible en https://www.uniboyaca.edu.co/es/centro-informacion/ documentos

Universidad de Boyacá. Rectoría. (marzo 21 de 2013b). Modelo Pedagógico. [Acuerdo No. 64]. Disponible en https://www.uniboyaca.edu.co/es/centro-informacion/documentos

Universidad del Magdalena. Vicerrectoría de extensión y Proyección Social. (2014). Informe de seguimiento a recién graduados se la universidad del magdalena 2011-2012. Santa Marta: Centro de egresados. Disponible en: https://www.unimagdalena.edu.co/Content/ Public/Docs/Entrada_Direcci\%C3\%B3n17/adjunto_1019-20180917172904_41.pdf

Villa, A. y Poblete, M. (2007). Aprendizaje basado en competencias: una propuesta para la evaluación de las competencias genéricas. Bilbao: Mensajero. Recuperado de http://biblio.upmx.mx/textos/14633.pdf

Andrés Correal Cuervo es Ingeniero de Sistemas con Master of Science in Telecommunications y Master en Seguridad de la Información Empresarial. Doctorando en Política y Gestión de la Educación. 25 años de experiencia en el sector profesional y académico, director de tecnología, decano, vicerrector y rector de la Universidad de Boyacá (Colombia). Presidente del comité departamental de tecnología de información de Boyacá. Par académico CNA. https://orcid.org/0000-0003-1856-3242

Ángela Carolina Bernal Álvarez es Administradora de Negocios Internacionales y Especialista en Gerencia de Mercadeo, con magister en Gerencia de Proyectos de la Universidad de Boyacá (Colombia). Con experiencia en líneas de trabajo relacionadas con el desempeño laboral de los egresados de instituciones de educación superior. https://orcid.org/0000-0001$7005-6745$

Juan Sebastián Cely Bottía es Ingeniero Industrial y Especialista en Ingeniería de Producción y Operaciones, con Maestría en Industria. Investigador, se desempeña como profesional de la División de Egresados he participado en la realización del "Estudio de Seguimiento a Egresados". https://orcid.org/0000-0003-0377-0935

Cristian Alejandro Aguilar Tovar es Ingeniero Industrial, maestrante en gestión humana y desarrollo organizacional. Profesional con competencias investigativas relacionadas con liderazgo, capacidad de análisis, búsqueda e interpretación de información necesarias para el planteamiento y desarrollo de proceso investigativos. https://orcid.org/0000-0001-6103-881X

Sandra Patricia Corredor Gamba es profesional en Terapia Respiratoria con Magister en Actividad Física. Docente investigadora del Grupo OXIGENAR, profesional de la División de egresados de la Universidad de Boyacá, ejecutando proyectos investigativos con base en seguimiento e impacto de egresados y opinión de empleadores. https://orcid.org/0000-00021382-0986 